DOI: 10.2478/romneu-2014-0016

\title{
Spinal meningiomas. Personal experience and review of literature
}

\author{
G. Iacob
}

Neurosurgery Clinic, Universitary Hospital, Bucharest, Romania

\begin{abstract}
Background: to present personal experience in the surgical management of spinal meningiomas, also the literature results review too.
\end{abstract}

Methods: 32 patients (4 men and 28 women) harboring spinal meningiomas who had undergone microsurgical resection were treated between 2002 and 2012 in our department. Clinical presentation, diagnosis, histological examination, microsurgical resection, functional outcome were evaluated, defining potential prognosis factors associated with these lesions.

Results: tumors site was intradural, extramedullary with different topography: the most common the thoracic region, posterolateral and antero-lateral. In all cases neurologic improvement was noted after operation, without instability, despite the extent of preoperative deficits. Transient motor deficits were observed in 2 thoracal anterior placed tumors without mortality. In 2 cases with semimaligne meningioma $(6,25 \%)$ recurrence was noted at one and two years after first operation, initial diagnosis was transitional type meningioma.

Conclusion: benign spinal meningiomas should have always early diagnosis and microsurgical total resection for a good outcome. For semimalignant or even malignant cases, radiotherapy should be considered.

Key words: spinal cord meningioma, microsurgical resection, functional outcome, recurrence.

\section{Introduction}

Spinal meningiomas occur after the fourth decade of life, over $70 \%$ of the patients are between the ages of 40 and 70 years with a mean age of 50 years, with similar frequency as the nerve sheat tumors, representing approximately $25 \%$ of all spinal cord tumors, $40 \%$ of intradural extramedullary tumors, without invading pia mater (1-8). Most meningiomas have a significant predilection for females $75 \%$ - $85 \%$ of cases; arising primarily in the thoracic region approximately $80 \%$; the cervical region is affected less often; lumbar and sacral tumors are relatively rare (9). Meningiomas typically grow slowly and usually with benign character, with a region of dural attachment, often seen dorsal-lateral, in a globoid configuration; rarely "en plaque meningiomas" - as a carpetlike (10)(11). Clinical findings variate from 
mild to significant neurologic dysfunction; the most frequent clinical findings are back pain, sensori-motor deficit and sphincter dysfunction (9). The MRI study of the entire neuraxis (12), with and without gadolinium enhancement, using both $\mathrm{T} 1$ and $\mathrm{T} 2$ weighted images, in sagittal and axial planes, was done in all cases, usefull for early diagnosis, operative planning and long term follow up. For a better prognosis, advances in microsurgery, ultrasonic dissection, peroperative monitoring should be used, since total surgical removal is the treatment of choice, generally curative, even when preoperative neurological status is poor (5)(9)(13). In this retrospective study I report my personal experience and literature data concerning this pathology.

\section{Methods}

In this retrospective study we include 32 patients (4 men and 28 women) who had undergone microsurgical resection between 2002 and 2012 in our department, harboring only spinal meningiomas (cranio-cervical meningiomas with intracranial extension were excluded). All these patients were examined preoperatively including: age - the mean age was 54,7 years (range $34-82$ years), the mean duration of symptoms was 13,7 months, scoring of motor weakness (no patient presented with paraplegia), sensory deficits, pain: severe pain or dysesthetic syndrome impairing patient's quality of life, dysesthesias, mild to moderate gait difficulty, bladder and bowel function. All patients were preoperative assessed by magnetic resonance imaging (MRI) of the spine with injection of gadolinium: spinal levels were determined using sagittal T1 or T2 sequences; topography, tumor insertion were assessed on axial sequences, computed tomography (CT) and electromyography (EMG). Neuroimaging studies evaluate: tumor location, cord edema, extent of spinal cord compression, site of dural attachment and calcification.

We used on the day of hospital admission, intravenous dexamethasone, furosemid, antalgic drugs continued for 5 days postoperatively up to patients discharge. After informed consent of the patient was signed, operation was done the day after hospital admission. All meningiomas were operated using a microsurgical technique via a posterior approach with the goal of spinal cord decompression. Antibiotic prophylaxy was done only in the operation day. Anesthesia was maintained with continuous propofol infusion $(20 \mathrm{ml} / \mathrm{h})$. No muscle relaxants were used after induction and intubation. ECG, pulse oximetry, invasive blood pressure, temperature, end-tidal carbon dioxide concentration, were monitored. The patients were positioned prone. After careful preoperative planning and radiologic intraoperatory control, a midline skin incision was performed extending two levels above and below the extent of the lesion. A monosegmental or multisegmental laminectomy above and below the extent of the tumor was performed, completed with partial facetectomy on tumor side in order to increase the viewing angle in only two cases. Dura was open longitudinal under operating microscope and fixed to the sides with moderate tension in order to expose, assure 
DOI: 10.2478/romneu-2014-0016

hemostasis, avoidind motor deficits. Arachnoid - sometimes with calcifications was open, dentate ligament was sectioned. On inspection tumor and spinal cord vascularisation are identified. Dura mater on tumor side was gently handled in order to identify lateral tumor attachments and tumor debulking starts using sharp dissection using a microsurgical technique and minimal bipolar electrocoagulation in order to avoid thermal and mechanical injury to the spinal cord. After tumor completely removal and careful hemostasis, the dura is coagulated in all cases and primarily closed in a watertight manner. No spinal stabilization was used. The pathologic examination was reported in all cases. The mean follow-up was 24 months; referring to clinical control postoperatively immediate after operation, at discharge; clinical and MRI control 1 months postoperatively and 1 , respectively 2 years after operation.

\section{Results}

Tumors site was thoracal, intradural, extramedullary, most common, posterolateral 22 cases, antero-lateral 4 cases, anterior to the spinal cord 2 cases; cervical intradural, extramedullary, postero-lateral 3 cases, anterior to the spinal cord 1 case. Mean tumor size was 35/30/25 mm. The most common presenting symptom was motor and sensory deficits, back pain, unsteady gait, sphincter dysfunction, whereas no patient presented with paraplegia. All meningiomas were operated using a microsurgical technique via a posterior approach, with complete tumor excision. Histopathology revealed the presence of meningiomas WHO grade I lesions: meningotheliomatous type 26 cases, psammomatous type 2 cases, transitional type 2 cases, fibrous 1 case, microcystic 1 case. In all cases resection was complete according to Simpson (despite Simpson's score, frequently used since 1957 for intracranial meningiomas), is not validated in spinal meningiomas: grade I in 26 cases, grade II in 6 cases. Transient motor deficits were observed in 2 thoracal anterior placed tumors without mortality. In two patients I have had a CSF leak. Postoperative all patients had marked neurological improvement, without instability. After a mean follow-up period of 24 months, only in 2 cases $(6,25 \%)$ despite initial diagnosis was transitional type meningioma, recurrence were noted, one and two years after first operation - histopathological diagnosis was semimaligne meningioma.

\section{Illustrative Case}

A 44-years-old woman presented with a 2years history of cervical and right shoulder pain, a 2-week history of progressive upper and lower right paresthesias, followed by mild right brahial weakness. Neurological examination revealed mild hypoesthesias in the right $\mathrm{C} 5-\mathrm{C} 8$ and left $\mathrm{C} 6-\mathrm{C} 8$ dermatomes; motor deficits $\mathrm{C} 5-\mathrm{C} 7$, right more than left, ASIA 3 grade; right Babinski and mild amiotrophy involving tenar emminence and interosseous right muscles. The patient underwent spinal MR examinations pre and postoperative $\mathrm{MR}$ imaging in a 1,5-Tesla MR system (General Electric). Sagittal T1 and T2 weighted images and axial T2 - weighted MR images of the cervical spine revealed an 
unique, right antero-laterally located intradural, extramedullary space-occupying lesion at the C2-C3 level; without extradural component; the lesion was hypointense on $\mathrm{T} 1$ - weighted images, hyperintense on T2weighted images and presented intense enhancement (figures 1-3).

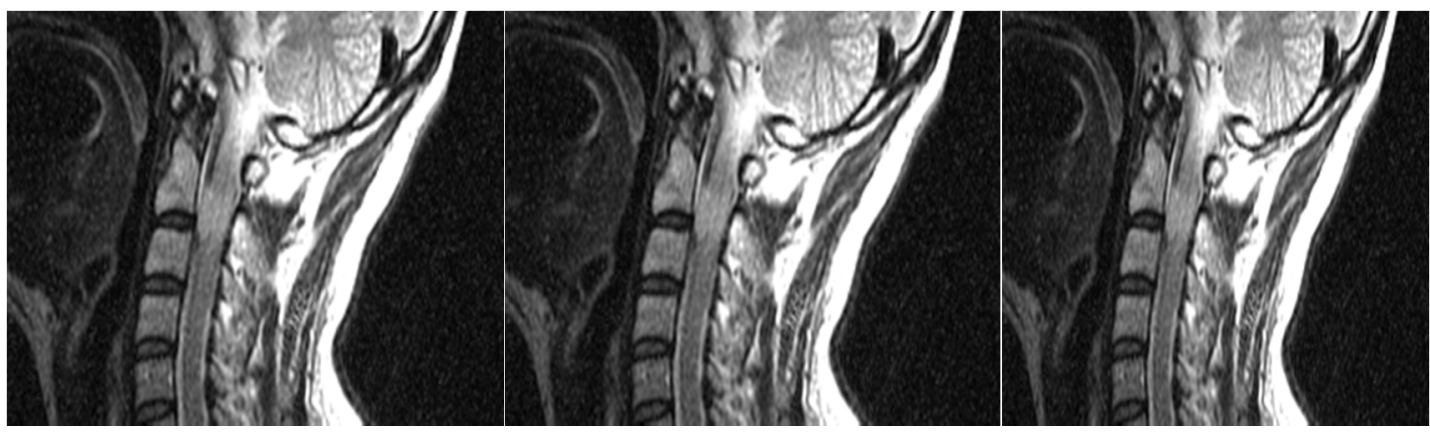

Figure 1 - Preoperative MRI: sagittal T2w FSE sequences reveals intradural extramedular tumor at C2 - C3 level with homogene hyperintense T2w signal

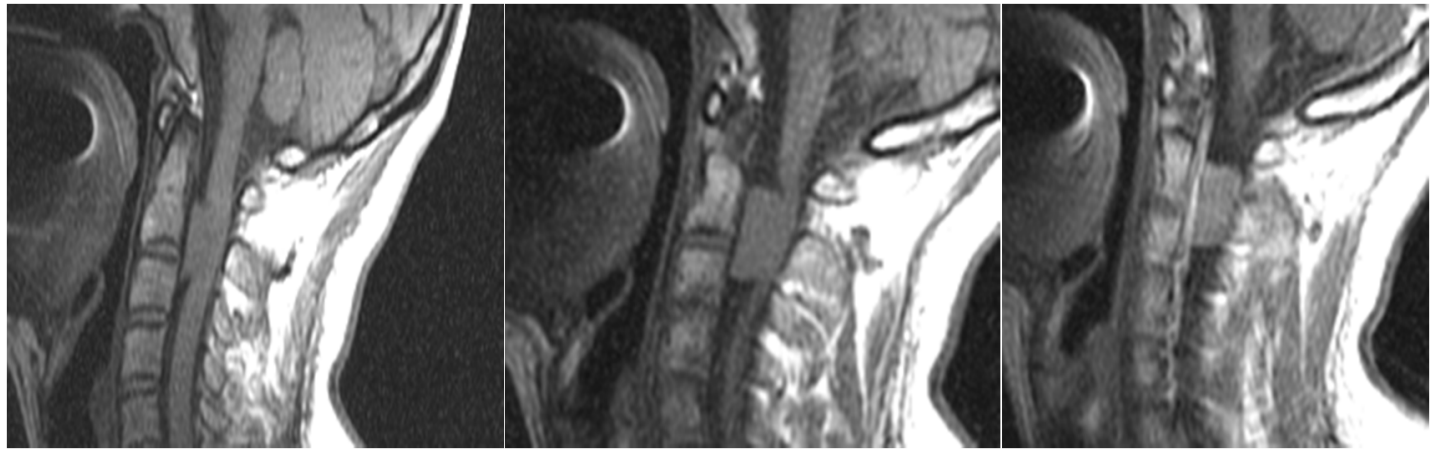

Figure 2 - Preoperative MRI: sagittal T1w sequences without and with paramagnetic contrast media reveals the tisular nature and the homogenous enhancement of the tumor

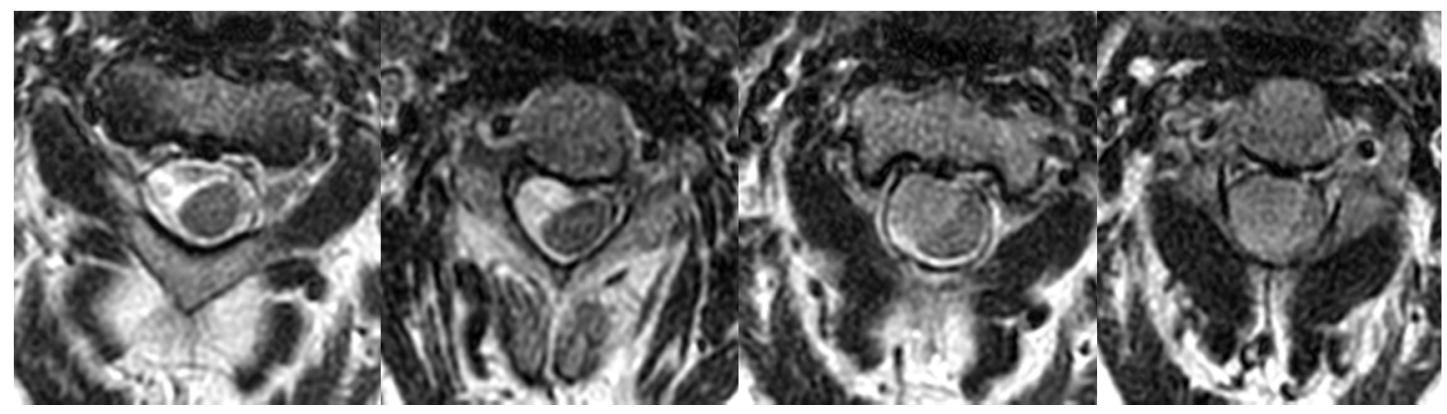

Figure 3 - Preoperative MRI: axial T2w sequences at the tumor level 
After induction of general anesthesia, the patient was positioned prone, on chest rolls, with head fixed in three point Mayfield head holder. Using a posterior approach, the posterior arch of $\mathrm{C} 1$, the lamina $\mathrm{C} 2$ and $\mathrm{C} 3$ were exposed and removed, without extending bony resection to the right transverse foramen C1-C3, allowing excellent visualisation of the tumor and ventral dura well past the midline. Dura was opened in a rostral-to-caudal direction, preserving the arachnoid and tacked laterally using 4-0 sutures to maximize exposure, disposing Gelfoam and cotton surgical strips laterally to assure hemostasis by venous bleeding. The arachnoid was then opened, dissected off the tumor and adjacent cord. We disclosed a $3 \times 2 \mathrm{~cm}$, well demarcated, red, intradural, unhomogenous, bleeding mass, located antero-lateral, displacing the spinal cord. Tumor was adherent and displaced both anterior and posterior right roots $\mathrm{C} 2-\mathrm{C} 3$ : also tumor was inserted on the dura mater anterior. Using the operating microscope, the arachnoid was opened and tumor was dissected, gradually debulked along the lateral aspect of the spinal canal and gently extracted from the anterolateral dural attachments. All nerve roots were preserved and the mass was completely removed. No methods of spinal reconstruction and instrumentation were used. (Figure 4)

Histopathological analysis of the resected tumor was compatible with the diagnosis of meningothelial meningioma. On microscopy (figure 5), specific features are seen.
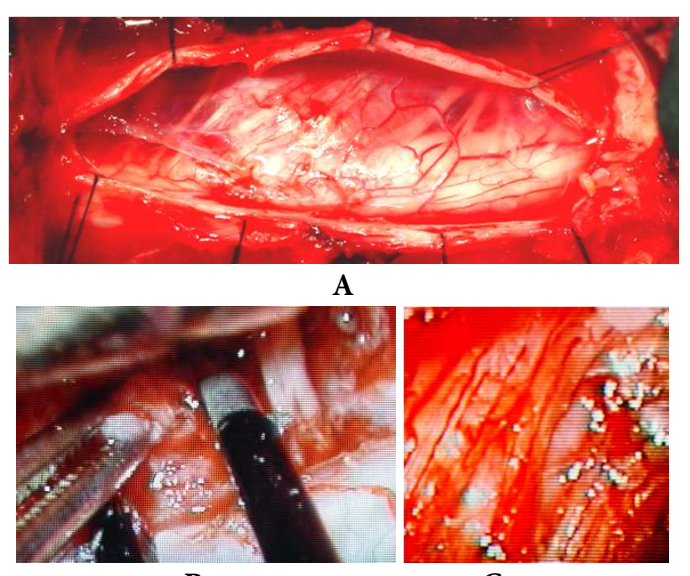

B

C

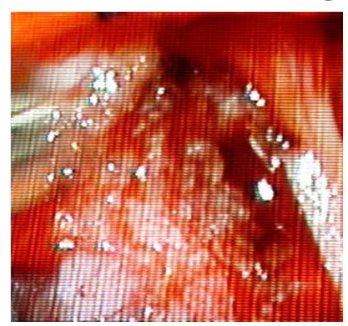

D

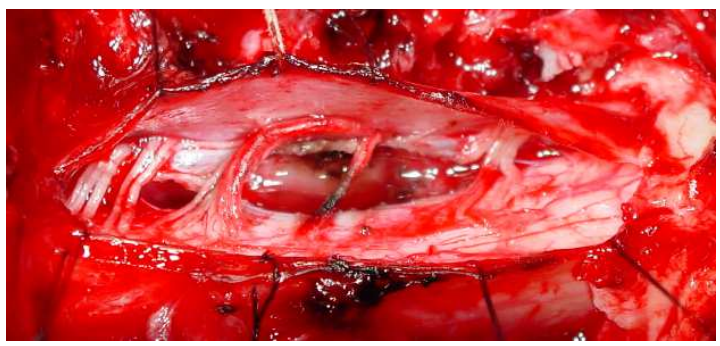

E

Figure 4 - Intraoperative photographies: A right antero-laterally placed, cervical meningioma, displacing the spinal cord laterally and anteriorly. Dura was opened, tacked laterally using 4-0 sutures and arachnoid was opened, dissected off the tumor and adjacent cord, B-D intraoperative photographies during tumor removal, E postoperative photography taken after removal of a cervical meningioma, without cutting right cervical roots 


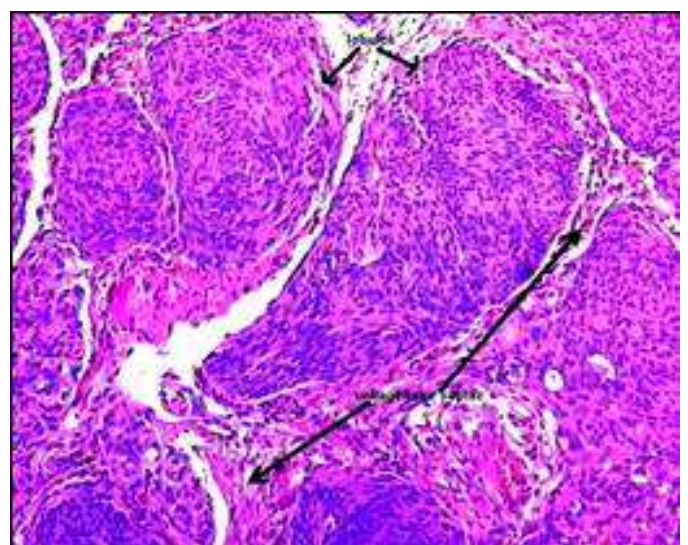

A

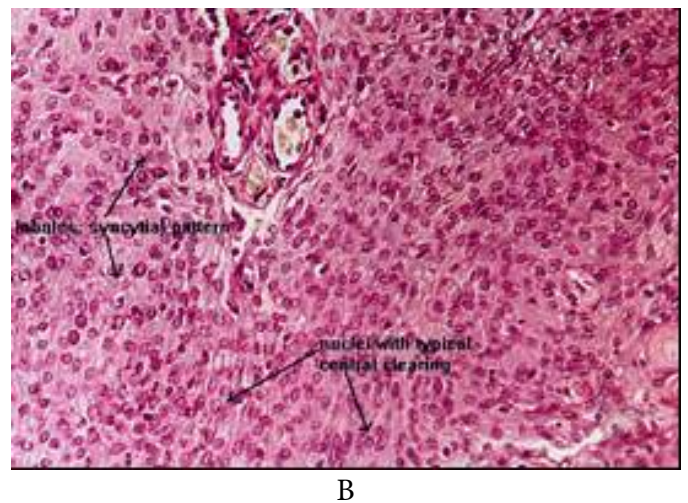

B

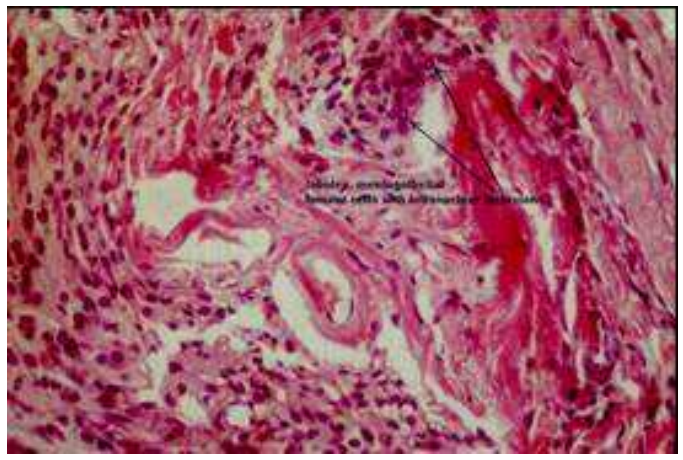

C

Figure 5 - Meningothelial meningioma microscopy photographies:

A.H\&E staining, X 100: tumour cells form lobules surrounded by thin collagenous septae

B.H\&E staining, X 200: solid areas of cells with poorly defined cell membranes (syncytial appeareance); like normal arachnoid, tumour cells are uniform, with oval or round nuclei with pale central clearing (slight tendency for the chromatin to be marginated at the periphery)

C.H\&E staining, X 200: perilobular collagen and reticulin are variable; usually nodular vascular thickening are seen
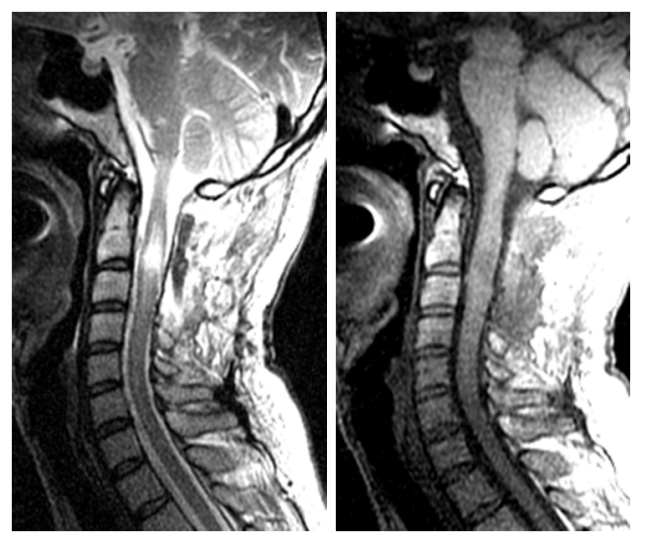

Figure 6 - Postoperative MRI: A sagittal T2 weighted image with no enhancement sugestive for local spinal cord oedema; B sagittal T1 image: a local postoperative oedema

The postoperative clinical course was uneventful; the patient was encouraged to begin ambulation the following morning and his neurological condition remarkably improved: sensory and motor disturbances resolved 1 month after the operation. A 4th day postoperative MRI showed local spinal cord oedema on T2 and T1 - weighted images with no enhancement sugestive for a restant tumor (Figure 6).

\section{Discussion}

In 1887, Sir Victor Horsley and Sir William Gowers (14) are credited with the first successful surgical removal of a spinal meningioma. In 1938, Cushing and Eisenhardt (15) defined the removal of a spinal 
DOI: 10.2478/romneu-2014-0016

meningioma as "one the most gratifying of all operative procedures". This affirmation was related both to the tendency of these tumors to develop in the lateral or postero-lateral surface of the spinal cord and to their extraaxial development, which renders spinal meningiomas easily dissectable from the spinal cord. Spinal meningiomas are usually solitary - $98 \%$, represent $25 \%$ to $46 \%$ of all primary spinal cord tumors, less frequently than intracranial ones and account for approximately $7.5-12.7 \%$ of all meningiomas (1), with a peak incidence in the fifth and sixth decades; similar as in my series; the mean age was 54,7 years (range 34-82 years)(2- 8)(16). The second most common, spinal, intradural, extramedullary tumors after the nerve sheath tumors; spinal meningiomas typically do not disrupt the pia, are infrequently adherent to the spinal cord and can be resect safely (17). Occasionally, spinal meningiomas grow in a sheet-like or collar-like manner around the spinal cord, infiltrating the pia mater (18). Multiple spinal meningiomas can occur rarely: 1 to $2 \%$, most often associated with neurofibromatosis type 2 , especially in the pediatric population, unrelated with neurofibromatosis (19)(20) or after Tamoxifen, which is a non-steroidal mixed estrogen drug widely used for patients with metastatic and primary breast cancer; utilized in the treatment of malignant gliomas with some efficacy (21).

There is a female predominance in the adult population: female to male ratio is $2: 1$ in intracranial meningiomas and 9:1 for spinal meningiomas, no sex predilection in children (22) (23). In my present series, the female/male ratio was 7: 1. Female predominance could be explained by hormonal factors as evidenced by progesterone and estrogen receptors frequently found on histological examination, as well as the reports of an association between meningioma and breast cancer or tumor growth and hormonal phases: pregnancy, menopause (24). Pregnancy frequently increase their size, explained by the discovery that a high percentage of meningiomas have progesterone receptors, whereas only a few have estrogen receptors. Several other receptor types have been found in some meningiomas, including receptors for androgens, insulin-like growth factor 1, epidermal growth factor, somatostatin, and dopamine. Other predisposing factors, primarily evaluated in cases of cranial meningiomas, may contribute to meningioma formation, although their roles appear to be minimal or controversial: previous radiation therapy, trauma and exposure to papovavirus, SV40, BK or herpes viruses (23).

The presumed site of origin of spinal meningiomas from arachnoidal cap cells located in the leptomeninges at the spinal nerve root exit zones, adiacent to the denticulate ligaments or entry zones of arteries in the spinal canal; but also from meningothelial cells making up the arachnoid villi near the dorsal root ganglia explaining why these tumors frequently arise in a lateral location to the spinal cord (25). The most common topography is the thoracic spine $80 \%$, (laterally $45-71 \%$, postero-laterally $10-$ $31 \%$ or antero-laterally to the spinal cord $15-$ 27\%) as in my series (1) (3). Cervical 
meningiomas (9) have a tendency to be more antero-lateral to the spinal cord $15 \%$, than thoracic ones, especially in patients younger than 50 years of age, representing a true surgical challenge and uncommon on the lumbo-sacral spine $2-10 \%$ (3). Spinal meningiomas usually are benign tumors with a capsule, typically grow in a globoid configuration with a region of dural attachment. They are classically found in an intradural extramedullary location, but can be extradural (10)(16)(26); purely extradural tumours account for only 3.5-7.0\% of all spinal meningiomas and occur more commonly in children than in adults or both extra- and intradural components mimiking on a dumbbell appearance in $5 \%$ of cases (27). There is also a very rare variety of spinal meningioma called "en plaque" (first spinal case was reported by Friedberg in 1972 (10)(11); term referring to "flat spreading carpets of tumor" Cushing and Eisenhardt (15) These variety are different from classic meningiomas: extensive tumor base, infiltration of surrounding structures, not respect tissue planes, ossifications, difficult to resect completely; also more likely to cause spinal arachnoiditis (18). Intramedullary meningiomas are exceedingly rare; only four cases have been reported to date (23). Genetic studies has found in meningiomas a translocation involving the area of chromosome 22, also known to harbor the gene responsible for neurofibromatosis type 2 (as like as in sporadic unilateral acoustic neuromas, sporadic spinal schwannomas and ependymomas). The abnormal loci for the meningioma and NF-2 genes are both on the distal end of the myoglobin locus of chromosome 22, but in separate regions. The proximity of these loci may explain the frequent association of the two tumors. Several oncogenes (gene sequences that actively induce tumor formation) also have been found in meningiomas: DNA coding for the Ha-ras, C-mos, myc, C-erb B and six oncogenes. Based on deletions on chromosome 22 , there is a two to fourfold increase in the rate of meningiomas seen in women with breast cancer as compared with age-matched control subjects (23).

Clinical findings in spinal meningiomas variate from mild to significant neurologic dysfunction, with many patterns of clinical presentation depending on: tumor location, the rate of tumor growth - onset and development are insidious, generally with a long clinical history until a diagnosis is made; in my present series was 13,7 months and correlates well with the time reported in the literature 12-24 months (9); dimension (usually they are small), tumor aggressiveness, the extent of spinal cord compression, myelopathy with vascular compromise with sensorimotor deficits, hyperactive deep tendon reflexes, Babinski sign, gait ataxia and weakness and the least common local or radicular pain, sphincter disturbances (2)(5)(12)(17). For cervical meningioma clinical findings are insidiously installed, asymmetric, occasionally remitting: occipital headache, neck pain; radicular symptoms $20 \%$ of patients, progression of motor and sensory deficits starting in one arm, greater ipsilateral to the side of the lesion and gradually involving the other extremities up to progressive tetraparesis with spasticity, 
DOI: 10.2478/romneu-2014-0016

atrophy and clumsiness of the hands, pathological reflexes, urinary incontinence $15 \%$ of patients; because most cervical meningiomas are located laterally or anterolaterally, displacing the spinal cord and the anterior spinal artery $(23)(26)$. In younger patients, they might have an aggressive clinical course (3).

The current standard diagnostic study for a spinal meningioma is MRI of the entire neuraxis (in order to reveal tumor in other locations) with and without gadolinium enhancement, using both T1 and T2 weighted images, in sagittal and axial planes, usefull for early diagnosis, operative planning, improving outcome and long term follow (12) (28-31). MRI study can delineate: tumor location and extensions vertically and laterally, extradural components, relation to the dura (site of dural attachment), spinal cord, vertebral artery (especially in the case of high cervical and foramen magnum tumors); presence of cord edema, intratumoral signal changes such as necrosis, hematoma or calcification. In general, spinal meningiomas are well circumscribed with dural tail sign and broad base attachment; usually isointense to the spinal cord ( $\mathrm{T} 1$ weighted images: isointense to slightly hypointense, possible heterogenous texture and $\mathrm{T} 2$ weighted images: isointense to slightly hyperintense with similar signal characteristics to intracranial meningiomas). $15 \%$ of patients with calcified meningiomas are hypointense on $\mathrm{T} 1$ and $\mathrm{T} 2$; $\mathrm{T} 1$ weighted images + Gadolinium: immediate and moderate homogeneous enhancement or only minimal contrast enhancement (6) (32). Cervical meningiomas are located intradurally, most frequently antero-laterally, without extension into the neural foramen (23). They are: iso or hypointense in relation to the spinal cord on the unenhanced T1weighted images, slightly hiperintense on T2 weighted images, with homogeneously enhance after the intravenous administration of gadolinium. There is no pathognomonic picture on MR images for atypical and invasive spinal meningiomas: heterogeneously enhancement or in a ring-like fashion, solitary or multiple lesions (32). In limited cases, threedimensional computed tomography-high resolution/myelography is indicated alone or in addition to MR imaging: see contraindications for $\mathrm{MR}$ imaging, bone destruction of osseous structures. Spinal CT indicate calcifications, isodense or moderately hyperdense mass, hyperostosis less common as in the intracranial forms. The main differential diagnosis of spinal meningiomas includes intradural extramedullary schwannomas. Statistically significant predictors of meningioma are: female, thoracic location - especially postero-lateral, calcification, dural tail, broad dural contrast, lack of foraminal extension, widening (27). Distinctions for a diagnosis of schwannoma or neurofibroma include: lumbar location, relation to the nerve root, widening of neural foramen, fluid signal intensity on T2 weighted images, rim enhancement on $\mathrm{MR}$ and scalloping on CT, not associated with a broad dural base (6)(30). Angiography can reveal a tumor blush with pathological vessels or early venous drainage. Plain $\mathrm{x}$-rays is usually normal, occasionally show bone erosions, fine calcifications within the spinal canal in only 2 
to $5 \%$ of cases with higher frequency in the en plaque type; these studies are usefull for differential diagnosis when bony abnormalities are consistent with metastatic diseases or neural foraminal enlargement suggest the diagnosis of nerve sheath tumor (3). For tetraparetic patients with cervical meningiomas, preoperative pulmonary function tests are mandatory (23).

Surgery is the treatment of choice, generally curative, suitable in the majority of the cases, even in patients with severe preoperative neurological deficits or advanced age, the goal of treatment is total surgical removal, including radical removal of their dural base without causing spinal instability and restoration of normal neurological function (4)(5)(9)(33-36). To facilitate tumor removal and diminish the risk of intraoperative spinal cord damage, at surgery: general anesthesia, high-dose corticosteroids, the operating microscope, the irrigating bipolar forceps to minimize heat transfer to the spinal cord, ultrasonic dissection, peroperative monitoring somato-sensoryevoked potential (SSEP) - easily recordable without adjusting the anesthetic regimen, transcranial motor-evoked potentials (TcMEPs) and continuous free running electromyography (EMG) - evaluate the pyramidal motor pathways, giving an immediate and conclusive feedback of motor tract integrity should be used (35). Generally surgical steps are: safe approach, gentle spinal cord decompression without rotation, by gradual tumor debulking, tumor capsule dissection with progressively removal. To improve Simpson resection grade (obtaining a
Simpson Grade I resection still is a desiderate, affecting recurrence rate and survival), even for calcified meningiomas "en plaque", dural attachment is in most reported studies: coagulated, separated: the inner layer is resected together with the tumor, preservind the outer layer or resection of the dural attachment with suturing of a patchgraft. However, in large series, there is no significant superiority of dural base resection over patients with dural base coagulation because recurrence is wery low (36)(41).

Alternative approaches are:

-posterior approach: more convenient, less invasive, technically more easy for dorsally placed tumors who can be removed totally, well-tolerated especially in older patients, allow wide enough exposure of the tumor and the dural attachment, even for those large tumors, use a standard hemilaminectomy, laminectomy or laminoplasty - postero-lateral approach (33), at one or two levels, with lateral extension even for anterior and antero-lateral meningiomas (35) if intraoperative monitoring are used! (transcranial motorevoked potentials (TcMEPs), somatosensoryevoked potential (SSEP) and free running electromyography (EMG), with fewer potential complications, eliminate the necessity of vertebrectomy; is facilitating complete tumor excision with good results. Posterior approach disadvantages are: limited space to expose the tumor and the need for spinal cord manipulation

-anterior approach indicated in anterior cervical meningioma or by a transthoracic approach for anterior spinal thoracic menigiomas (33)(36-39). Such approach has 
DOI: 10.2478/romneu-2014-0016

many advantages: large bony window of access through a corpectomy, good tumor visualization in front of the spinal cord, good control over bleeding during meningioma resection, no spinal cord manipulation during meningioma resection, preserving anterior spinal artery and nerve roots vessels. Anterior approach disadvantages are: time consuming, generate the necessity to fixation-fusion of the spine both anterior on several levels and sometimes posterior (posterior fixation is recommended by many authors after three levels of corpectomy)(40), cerebrospinal fluid leakage which could be avoided by watertight dural closure, application of fibrin glue, filling the dead space with muscle fascia and prophylactic lumbar CSF drainage (49).

For high cervical spinal meningiomasfrom the C3 cephalad (23) special considerations are made:

- surgical indication must be reserved to patients with signs or symptoms of spinal cord compression installed rapidely in spite of corticosteroid administration. For the asymptomatic patient or for patients with NF1 and multiple tumors, a closed followed up conservatively with serial MRIs should be performed.

- high cervical meningiomas tend to be intradural, entirely extradural or have both intra-and extradural components.

-tumors located posteriorly or posterolateral to the spinal cord are approached through the postero-lateral approach, a lateral extension of the standard midline posterior approach. These technique enables the approach of most intradural extramedullary tumors, enlargement toward the opposite side for those located more anteriorly, especially for large tumors and may offer controll to both vertebral arteries, especially in high cervical meningiomas with extradural component, where to achieve a complete resection, the vertebral artery should be mobilized.

-tumors placed ventral or ventro-lateral to the spinal cord are removed through a straight forward anterior approach, providing a wide field, also a good dural closure.

- after total removal, significant neurological improvements are usually observed, whereas tumor recurrence rate has been reported to be $1.3-14 \%$.

Spinal meningioma are histopathologically usually benign (more than $95 \%$ being classified as WHO grade I lesions); are slowly growing tumors, without pia mater invasionimportant anatomical detail, which improves the ability to resect them safely. Meningotheliomatous, Roux et al. $44 \%$ in their series (41) and psammomatous subtypes (20\%) are the most common, similar to my series; also immunohistochemical staining positive for vimentin and epithelial membrane antigen, progesterone receptor activity was found frequently Another histological subtypes cited in the literature are: fibroblastic, chordoid, transitional, vacuolated, clear cell, but also atypical or WHO grade II, malignant or WHO grade III, meningosarcoma (32). In younger patients, meningiomas grow in a more aggressive pattern, especially in cervical topography, frequently with an extradural component, are very invasive, angioblastic, involving the bone and cervical soft tissue, including the vertebral artery. There are not valid data about the rate of atypical and 
malignant meningioma in the spinal canal. Despite Ki-67 index value, known as predictor of intracranial meningiomas for recurrence and overall survival, for spinal meningiomas, the value of this index in predicting behavior has not been yet fully elucidated and further studies are needed (42)(43). Comparing spinal and intracranial meningiomas the proliferation rates of intracranial meningiomas were significant higher (Ki-67 $3.6 \%$ versus spinal Ki-67 of $2.48 \%$ )

Particular situation are leated to spinal en plaque meningioma, who may not always be totally resectable because this variety tends to invade the arachnoid layer (18)(27) see Caroli et al. (11) in only 3 of the patients from a series of 7 cases of en plaque spinal meningiomas. For partially resected tumors recurrence is the rule. Similar in patients with heavily calcified meningiomas with a ventral or ventro-lateral location, a partial vertebrectomy and/or costotransversectomy approach allows safer tumor manipulation and removal, with subsequent instrumentation; especially those tumors occurring at the cervico-thoracic and/or thoraco-lumbar junction, dorsal stabilization should be considered to prevent junctional kyphosis. Generally the prognosis of benign spinal meningioma with complete resection is very good, even when preoperative neurological status is poor; improvement are seen over $90 \%$ of the cases immediately or gradual up to 18 months, with a good long term functional outcome (2)(5)(9)(13). Spinal menigioma prognosis are more favorable compared to intracranial localizations: mean $6.2 \%$ for morbidity and $2.1 \%$ for mortality; related to the preoperative neurological status, the patient's age, the duration of symptoms, tumor location in the spinal canal: anterior or antero-lateral, total removal of the lesion, histological tumor grade, Simpson resection grade, invasion of pia mater (9)(44). Meningiomas located directly anteriorly, a low preoperative Karnofsky score, a short history, calcified tumor (45) with en plaque extension, cervical location with an extradural component (which are often more vascular and aggressive), tumor progression, invasion of the arachnoid/pia, arachnoid scarring (46); also reoperation for recurrent tumor may expose to transiently neurological worsening after surgery. Nevertheless even after successful surgery, a possible increase of the motor deficit or even permanent deficit (paraplegia) could be seen if spinal meningiomas are located anteriorly to the spinal cord. Klekamp J, Samii M.(46) found $11.2 \%$ of complication in 117 cases of spinal cord meningiomas and Solero et al. (47) found $3.5 \%$ in 174 cases; also sensory disturbances, dysesthesia syndrome progressing to myelopathy (46).

Recurrence after spinal meningioma resection has been reported to be $1.3-14 \%$ (4)(48); for meningiomas that have been totally resected is $1.3 \%$ at 5 years and $6 \%$ at 14 years, related to: subtotal resection, anterior location, calcification, malignancy proved histologically, multiplicity of lesions, young age. Recurrence in the patients with dural base coagulation was so low, that no significant superiority of dural base resection over dural base coagulation could be found (41).

Postoperatory complications which could be avoided by a meticulous technique (46)(49) 
DOI: 10.2478/romneu-2014-0016

are: CSF leak (2 cases in my series), pseudomeningocele development, wound infections, aseptic meningitis, arachnoiditis (as a result of blood left in the subarachnoid space or as a result of placement of dural substitutes of animal origin), syringomyelia, spinal destabilization, as well as other routine complications of spinal surgery and general anesthesia. Less frequent complications are pulmonary embolism, pneumonia,and myocardial infarction. pulmonary embolism.

In invasive and high-grade spinal meningiomas therapeutic alternatives are: extensive tumor resection, completed with radiosurgery (50) or stereotactic CyberKnife frameless stereotactic radiosurgery as a single fraction therapy (51)(52), combined with chemotherapy (as hydroxyurea), hormonal manipulation with tamoxifen (antiestrogen), mifepristone (antiprogesterone), recombinant interferon $a 2 b$.

\section{Conclusions}

My personal experience based on this retrospective study argue that even when tumor is late discovered and neurological compromise exist, in the high cervical area or anterior spine topography, spinal meningiomas can be successfully resected today with favorably outcomes, low complication rates, offering a better life quality. Early diagnosis by MRI, corticosteroids, microsurgery techniques, intraoperative electrophysiological monitoring are mandatory. For anterior spine approaches if an anterior approach is chosed, reconstruction and instrumentation methods should be used. In cases of semimalignant or malignant transformation, close follow-up and adjuvant therapies must be considered.

\section{Correspondence}

Dr. Luis Rafael Moscote. Universidad de

Cartagena, Cartagena de Indias, Colombia.

e-mail:mineurocirujano@aol.com

\section{References}

1. Albanese V, Platania N - Spinal intradural extramedullary tumors. Personal experience; J Neurosurg Sci 2002, 46:18-24

2. Gezen F, Kahraman S, Canakci Z - Review of 36 cases of spinal cord meningioma. Spine 2000, 25:727-731

3. Cohen-Gadol AA, Zikel OM, Koch CA - Spinal meningiomas in patients younger than 50 years of age: a 21-year experience, J Neurosurg (Spine) 2003, 98:258-263 4. Gottfried, O.N., et al. - Spinal meningiomas: surgical management and outcome, Neurosurg Focus 2003, 14 (6), e2

5. Morandi, X., Haegelen, C., et al., - Results in the operative treatment of elderly patients with spinal meningiomas. Spine 200429 (19), 2191-2194

6. De Verdelhan O, et al. - MR imaging features of spinal schwannomas and meningiomas; J.Neroradiol 2005, 32:42-49

7. Haegelen, C., et al. - Results of spinal meningioma surgery in patients with severe preoperative neurological deficits. Eur Spine 2005, J 14 (5), 440-444

8. Cavanaugh, D.A., et al. - Intraspinal meningioma in a 101-year-old: should age determine the aggressiveness of intervention?, Surg.Neurol. 2008, 69 (2), 130-134

9. Riad H., S. Knafo S. et al. - Spinal meningiomas: Surgical outcome and literature review, Neurochirurgie 2013, 59, 30-34

10.Achari G, et al. - Extradural meningioma en-plaque of the cervical cord, Neurol Res. 2000; 22: 551-553

11.Caroli E, Acqui M, Roperto R, et al. Spinal en-plaque meningiomas: a comtemprary experience. Neurosurgery. 2004; 55: 1275-1279

12.Saraceni, C., Harrop, J.S., - Spinal meningioma: chronicles of contemporary neurosurgical diagnosis and management, Clin Neurol Neurosurg 2009, 111 (3), 221226

13.Yoon, S.H., et al. - Surgical outcome of spinal canal 
meningiomas, J Korean Neurosurg Soc 2007, 42 (4), 3003004

14.Mulholland R.C. - Sir William Gowers 1845-1915, Spine 1996, 21: 1106-1110

15.Cushing H. \&Eisenhardt L. - Meningiomas: Their Clasification, Regional Behaviour, Life History and Surgical End Results, Springfield 1938, IL: Charles C. Thomas

16.Dagain, .A., et al. - Extradural spinal meningioma: case report. Neurochirurgie 2009, 55 (6), 565-568

17.Sandalcioglu, I.E., et al. - Spinal meningiomas: critical review of 131 surgically treated patients. Eur Spine J 2008, 17 (8), 1035-1041

18.Gamache F, Wang J, Deck M, Heise C - Unusual appearance of an en plaque meningioma of the cervical spinal canal. A case report and literature review, Spine 2001, 26(5): E87-E89

19.Silva J.A., Holanda M.M., et al. - Multiple meningiomas within the spinal canal: case report with 23 tumors, Arq Neuropsiquiatr.2005; 63: 166-170

20.Keskin F., Kalkan E., Karatas Y - A Case of Upper Thoracic Intradural-Extramedullary Multiple Meningiomas, Neurosurg Q 2013; 23: 224-225

21.Colazza G.B. et al.- Multiple spinal meningiomas after tamoxifen therapy: a case report, Neurol Sci 2003, 24: 3739

22.Peker S, et al. - Spinal meningiomas: evaluation of 41 patients. J NeurosurgSci 2005, 49:7-11

23.Cooper P.R., Wienecke R.J., White B.J. - Spinal Meningiomas, in Batjer H.H., Loftus Ch.M. - Textbook of Neurological Surgery, Lippincott Williams \& Wilkins, 2003, vol. II, 1857-1864.

24.Barnholtz-Sloan J.S., Kruchko C. - Meningiomas: causes and risk factors. Neurosurg Focus 2007, 23 (4), E2. 25.Setzer M., Vatter H., et al. - Management of spinal meningiomas: surgical results and a review of the literature, Neurosurg. Focus 2007, 23 (4): E14

26.Frank, B.L., et al. - Cervical extradural meningioma: case report and literature review. J Spinal Cord Med 2008, 31 (3), 302-305

27.Messori A., Rychlicki F., Salvolini U. - Spinal epidural en-plaque meningioma with an unusual pattern of calcification in 14-year-old girl: case report and review of the literature, Neuroradiology 2002, 44: 256-260

28.Abul-Kasim K, et al. - Intradural spinal tumors: current classification and MRI features. Neuroradiology 2008, 50:301-314
29.Alorainy I.A - Dural tail sign in spinal meningiomas, Eur J Radiol. 2006, 60: 387-391

30.Gebauer G.P., Farjoodi P., et al. - Magnetic resonance imaging of spinal tumours: classification, differential diagnosis and spectrum of disease, J. Bone Jnt.Surg Am 2008, 90: 146-162

31.Lee J.Y. - Radiological findings of spinal schwannomas and meningiomas: focus on discrimination of two disease entities, Eur Radiol 2009, 19: $2707-2715$

32.Schaller, B., - Spinal meningioma: relationship between histological subtypes and surgical outcome? J Neurooncol 2005, 75 (2), 157-161

33.Gambardella G, Gervasio O, Zaccone C - Approaches and surgical results in the treatment of ventral thoracic meningiomas. Review of our experience with a posterolateral combined transpedicular-transarticular approach. ActaNeurochir (Wien) 2003, 145:385-392

34.Sacko O. et al. - Spinal meningioma surgery in elderly patients with paraplegia or severe paraparesis:a multicenter study. Neurosurgery 2009, 64:503-509

35.Voulgaris S. et al. - Posterior approach to ventrally located spinal meningiomas, Springer-Verlag 2010, Eur Spine J 2010, 19:1195-1199

36.Payer M. - The anterior approach to anterior cervical meningiomas: review illustrated by a case, ActaNeurochir (Wien) (2005) 147: 555-560

37.D'Aliberti G., Talamonti G., Villa F. - Anterior approach to thoracic and lumbar spine lesions: results in 145 consecutive cases. J Neurosurg Spine 2008, 9: 466-482 38.Banczerowski P., Lipoth L., Vajda J., Veres R. - Surgery of ventral intradural midline cervical spinal pathologies via anterior cervical approach: our experience. Ideggyogy Sz 2003, 56: 115-118

39.Brunon J., Fuentes J.M. - Anterior and antero-lateral surgery of the lower cervical spine (25 years after $\mathrm{H}$. Verbiest), 2: Indications, results, complications, Neurochirurgie 1996, 42(4-5): 229-248

40.Singh K, Vaccaro A, et al. - Biomechanical comparison of cervical spine reconstructive techniques after a multilevel corpectomy of the cervical spine. Spine 2003, 28(20): 2352-2358

41.Roux F.X, Nataf F., Pinaudeau M., et al. - Intraspinal meningiomas: review of 54 cases with discussion of poor prognosis factors and modern therapeutic management. Surg Neurol. 1996;46:458-464 
DOI: 10.2478/romneu-2014-0016

42.Bruna J, et al. - Ki-67 proliferative index predicts clinical outcome in patients with atypical or anaplastic meningioma. Neuropathology 2007, 27:114-120

43.Roser F, Nakamura M, Bellinzona M - Proliferation potential of spinal meningiomas. Eur Spine J 2006, 15:211-215

44.Pena M., Galasko C.S., Barrie J.L. - Delay in diagnosis of intradural spinal tumors. Spine 1992, 17: 1110-1116, 45.Naderi, S., et al., - Ossified thoracic spinal meningioma in childhood: a case report and review of the literature. Clin Neurol Neurosurg 2001, 103 (4), 247-249

46.Klekamp J, Samii M. - Surgical results for spinal meningiomas, Surg. Neurol. 1999; 52:552-562; also Klekamp J, Samii M. - Meningiomas, Surgery of the spinal Tumors, Springer 2007, 4.5.1., 248-260

47.Solero C.L., Fornari M., Giombini S., et al. - Spinal meningiomas: review of 174 operated cases.
Neurosurgery 1989; 125:153-160

48.Nadkarni, B., et al. - Recurrent spinal meningioma: a case report withreview of the literature. J OrthopSurg (Hong Kong) 2005, 13 (3), 326-329

49.Misra, S.N., - Avoidance of structural pitfalls in spinal meningioma resection. Neurosurg Focus 2003, 14 (6), e1 50.Gerszten P.C., Burton S.A., Ozhasoglu C. Radiosurgery for benign intradural spinal tumors, Neurosurgery 2008, 62:887-895

51.Robert L.D., Mi-Ryeong R. et al. - CyberKnife Radiosurgery for Benign Intradural Extramedullary Spinal Tumor, Neurosurgery, 2006, 58, 4, 674-685 52.Chang UK, Rhee $\mathrm{CH}$, et al. - Radiosurgery using the Cyberknife for benign spinal tumors: Korea Cancer Center Hospital experience. J Neuroonc. 2011 Jan; 101(1): 91-9 